\title{
Dual Bregman proximal methods for large-scale $0-1$ problems
}

\author{
K.C. Kiwiel \\ System Research Institute, Warsaw, Poland. \\ e-mail: kiwiel@ibspan.waw.pl \\ P.O. Lindberg, Andreas Nöu \\ Royal Institute of Technology, Stockholm, Sweden. \\ e-mail: pol@math.kth.se, andreasn@math.kth.se
}

\begin{abstract}
We describe an extension of the Bregman proximal method for convex programming, employing $B$-functions as generalizations of Bregman functions that cover more applications. We allow inexact subproblem solutions, increasing their accuracy successively to retain global convergence. Our framework is applied to Lagrangian relaxations of large-scale set covering problems that arise in airline crew scheduling.
\end{abstract}

\section{Keywords}

Proximal point methods, Bregman functions, set covering problems.

\section{INTRODUCTION}

We describe an extension of the Bregman proximal method for convex programming (Censor and Zenios, 1992; Chen and Teboulle, 1993; Eckstein, 1993). First, we consider $B$-functions of (Kiwiel, 1994) as generalizations of Bregman functions (Censor and Lent, 1981) that cover more applications. Second, we allow inexact subproblem solution$\mathrm{s}$, increasing their accuracy successively to retain global convergence. Our framework is applied to Lagrangian relaxations of large-scale set covering problems that arise in airline crew scheduling (Wedelin, 1993). We report encouraging preliminary computational experience.

Our notation is fairly standard. $\langle\cdot, \cdot\rangle$ and $|\cdot|$ are the Euclidean inner product and norm respectively. $\mathbb{R}_{+}$and $\mathbb{R}_{>}$are the nonnegative and positive reals respectively. $\operatorname{cl} C$ and ri $C$ denote the closure and relative interior of $C \subset \mathbb{R}^{n}$. We let ${ }^{{ }_{C}} C(x)=0$ if $x \in C$, $\infty$ otherwise, and $\imath_{C}^{*}=\sup _{x \in C}\langle\cdot, x\rangle$. For a convex function $f, \mathcal{D}_{f}=\{x: f(x)<\infty\}$, $\partial f(\cdot)=\{g: f(x) \geq f(\cdot)+\langle g, \cdot-x\rangle \forall x\}$ and $\mathcal{D}_{\partial f}=\{x: \partial f(x) \neq \emptyset\}$.

Research supported by the Swedish Transport and Communications Research Board, the Göran Gustafsson Foundation and the Polish State Committee for Scientific Research under Grant 8S50502206. 


\section{$2 \quad B$-FUNCTIONS}

For any convex function $h$ on $\mathbb{R}^{n}$, we define its difference functions

$D_{h}^{b}(x, y)=h(x)-h(y)-\imath_{\partial h(y)}^{*}(x-y) \quad \forall x, y \in \mathcal{D}_{h}$,

$D_{h}^{\sharp}(x, y)=h(x)-h(y)+\imath_{\partial h(y)}^{*}(y-x) \quad \forall x, y \in \mathcal{D}_{h}$.

By convexity, $h(x) \geq h(y)+\imath_{\partial h(y)}^{*}(x-y)$ and

$0 \leq D_{h}^{b}(x, y) \leq h(x)-h(y)-\langle g, x-y\rangle \leq D_{h}^{\sharp}(x, y) \quad \forall x, y \in \mathcal{D}_{h}, g \in \partial h(y)$.

$D_{h}^{\mathrm{b}}$ and $D_{h}^{\sharp}$ generalize the usual D-function of $h$ (Censor and Lent, 1981), defined by

$D_{h}(x, y)=h(x)-h(y)-\langle\nabla h(y), x-y\rangle \quad \forall x \in \mathcal{D}_{h}, y \in \mathcal{D}_{\nabla h}$,

since

$D_{h}(x, y)=D_{h}^{\mathrm{b}}(x, y)=D_{h}^{\sharp}(x, y) \quad \forall x \in \mathcal{D}_{h}, y \in \mathcal{D}_{\nabla h}$.

(Readers only interested in our applications may assume $h$ is s.t. $\partial h(y)=\{\nabla h(y)\}$ for all points $y$ of interest.)

Definition 1 (Kiwiel, 1994) A closed proper (possibly nondifferentiable) convex function $h$ is called a $B$-function (generalized Bregman function) if

(a) $h$ is strictly convex on $\mathcal{D}_{h}$.

(b) $h$ is continuous on $\mathcal{D}_{h}$.

(c) For every $\alpha \in \mathbb{R}$ and $x \in \mathcal{D}_{h}$, the set $\mathcal{L}_{h}^{b}(x, \alpha)=\left\{y \in \mathcal{D}_{\partial h}: D_{h}^{b}(x, y) \leq \alpha\right\}$ is bounded.

(d) For every $\alpha \in \mathbb{R}$ and $x \in \mathcal{D}_{h}$, if $\left\{y^{k}\right\} \subset \mathcal{L}_{h}^{b}(x, \alpha)$ is a convergent sequence with limit $y^{*} \in \mathcal{D}_{h} \backslash\{x\}$, then $D_{h}^{\sharp}\left(y^{*}, y^{k}\right) \rightarrow 0$.

General properties of $B$-functions are discussed by (Kiwiel, 1994). From lack of space, we only quote the following simple consequence of Lemmas 2.8-2.10 of (Kiwiel, 1994).

Lemma 2 Let $\psi$ be a proper convex function on $\mathbb{R}$. Then $h(x)=\sum_{i=1}^{n} \psi\left(x_{i}\right)$ is a $B$ function iff $\psi$ is closed, essentially strictly convex and $\mathcal{D}_{\psi^{*}}=$ ri $\mathcal{D}_{\psi^{*}}$, where $\psi^{*}(\cdot)=$ $\sup _{t}\langle\cdot, t\rangle-\psi(t)$ is the conjugate of $\psi$. In particular $\mathcal{D}_{\psi^{*}}=\operatorname{ri} \mathcal{D}_{\psi^{*}}=\mathbb{R}$ if $\mathcal{D}_{\psi}$ is bounded.

Examples 3 Let $\psi: \mathbb{R} \rightarrow(-\infty, \infty]$ and $h(x)=\sum_{i=1}^{n} \psi\left(x_{i}\right) ;$ then $h^{*}(y)=\sum_{i} \psi^{*}\left(y_{i}\right)$. In each example, $h$ is an essentially smooth $B$-function with $\partial h=\{\nabla h\}$ and $\mathcal{D}_{h^{*}}=\mathbb{R}^{n}$.

1 (Fermi/Dirac). $\psi(t)=t \ln t+(1-t) \ln (1-t)$ on $\mathcal{D}_{\psi}=[0,1](0 \ln 0=0)$. Then $\psi^{*}(t)=\ln (1+\exp t)$ on $\mathcal{D}_{\psi^{*}}=\mathbb{R}$.

2 (Burg's entropy). $\psi(t)=-\ln t-\ln (1-t)$ on $\mathcal{D}_{\psi}=[0,1]$. Then $\psi^{*}(t)=\frac{1}{2}\left[\left(t^{2}+4\right)^{1 / 2}+\right.$ $t-2]+\ln \left[\left(t^{2}+4\right)^{1 / 2}-2\right]-\ln t^{2}$ for $t \neq 0, \psi^{*}(0)=-\ln 4$.

3 (Hellinger) . $\psi(t)=-\sqrt{t(1-t)}$ on $\mathcal{D}_{\psi}=[0,1]$. Then $\psi^{*}(t)=\frac{1}{2}\left(t+\sqrt{1+t^{2}}\right)$. 


\section{AN INEXACT $B$-PROXIMAL METHOD}

Consider the convex minimization problem $f_{*}=\inf _{X} f$, where $f: \mathbb{R}^{n} \rightarrow(-\infty, \infty]$ is closed proper convex and $X$ is a nonempty closed convex set in $\mathbb{R}^{n}$. Suppose $h$ is a $B$ function s.t. $\mathcal{D}_{f_{X}} \cap \mathcal{D}_{h} \neq \emptyset$, where $f_{X}=f+\imath_{X}$ is the essential objective. Let $\left\{t_{k}\right\} \subset \mathbb{R}_{>}$ and $\left\{\epsilon_{k}\right\} \subset \mathbb{R}_{+}$satisfy $\sum_{k=1}^{\infty} t_{k}=\infty$ and $\lim _{l \rightarrow \infty} \sum_{k=1}^{l} t_{k} \epsilon_{k} / \sum_{k=1}^{l} t_{k}=0$.

The inexact Bregman-prox method of (Kiwiel, 1995) runs as follows. At iteration $k \geq 1$, having $x^{k} \in \mathcal{D}_{f_{X}} \cap \mathcal{D}_{\partial h}, \gamma^{k} \in \partial h\left(x^{k}\right)$ and $D_{h}^{k}\left(\cdot, x^{k}\right)=h(\cdot)-h\left(x^{k}\right)-\left\langle\gamma^{k}, \cdot-x^{k}\right\rangle$, minimize $f_{X}+D_{h}^{k}\left(\cdot, x^{k}\right) / t_{k}$ approximately to find $x^{k+1}, \gamma^{k+1}$ and $p^{k+1}$ satisfying.

$\gamma^{k+1} \in \partial h\left(x^{k+1}\right)$,

$t_{k} p^{k+1}+\gamma^{k+1}-\gamma^{k}=0$,

$p^{k+1} \in \partial_{\epsilon_{k}} f_{X}\left(x^{k+1}\right)$

$f_{X}\left(x^{k+1}\right) \leq f_{X}\left(x^{k}\right)$.

We note that (4)-(6) imply

$x^{k+1} \approx \arg \min \left\{f(x)+D_{h}^{k}\left(x, x^{k}\right) / t_{k}: x \in X\right\}$

with $0 \leq D_{h}^{b}\left(\cdot, x^{k}\right) \leq D_{h}^{k}\left(\cdot, x^{k}\right) \leq D_{h}^{\sharp}\left(\cdot, x^{k}\right)$; in fact $x^{k+1}$ is an $\epsilon_{k}$-minimizer of $f_{X}+$ $D_{h}^{k}\left(\cdot, x^{k}\right) / t_{k}$ (Kiwiel, 1995). The exact Bregman-prox method (Censor and Zenios, 1992; Chen and Teboulle, 1993; Eckstein, 1993) corresponds to assuming that $\epsilon_{k} \equiv 0$ and $\mathcal{D}_{\partial h}=\mathcal{D}_{\nabla h}$, so that $p^{k+1} \in \partial f_{X}\left(x^{k+1}\right), \gamma^{k}=\nabla h\left(x^{k}\right)$ and $D_{h}^{k}=D_{h}$ for all $k$ (cf. (3)). A particular implementation of our inexact method will be described in the next section.

The following global convergence results are established in (Kiwiel, 1995).

Theorem 4 (a) $f_{X}\left(x^{k}\right) \downarrow \inf _{\mathcal{D}_{h}} f_{X}=\inf _{\mathrm{cl}\left(\mathcal{D}_{h} \cap \mathcal{D}_{f X}\right)} f$. Hence $f_{X}\left(x^{k}\right) \downarrow \inf _{X} f$ if $\mathcal{D}_{f_{X}} \subset$ $\mathcal{D}_{h}$. If ri $\mathcal{D}_{h} \cap \operatorname{ri} \mathcal{D}_{f_{X}} \neq \emptyset$ (e.g. , int $\left.\mathcal{D}_{h} \cap \mathcal{D}_{f_{X}} \neq \emptyset\right)$ then $\inf _{\mathcal{D}_{h}} f_{X}=\inf _{\text {cl } \mathcal{D}_{h}} f_{X}=$ $\inf _{\left(\mathrm{cl} \mathcal{D}_{h}\right) \cap\left(\mathrm{cl} \mathcal{D}_{f_{X}}\right)} f$. If $\operatorname{ri}_{f_{X}} \subset \operatorname{cl} \mathcal{D}_{h}\left(e . g ., \mathcal{D}_{\partial f_{X}} \subset \operatorname{cl} \mathcal{D}_{h}\right)$ then $\operatorname{cl} \mathcal{D}_{h} \supset \operatorname{cl} \mathcal{D}_{f_{X}}$ and $\operatorname{Arg} \min _{X} f \subset \operatorname{cl} \mathcal{D}_{h}$.

(b) If $\sum_{k=1}^{\infty} t_{k} \epsilon_{k}<\infty$ and $X_{*}=\operatorname{Arg} \min _{\mathcal{D}_{h}} f_{X}$ is nonempty then $\left\{x^{k}\right\}$ converges to some $x^{\infty} \in X_{*}$, and $x^{\infty} \in \operatorname{Arg} \min _{X} f$ if $\mathcal{D}_{f_{X}} \subset \mathcal{D}_{h}$.

(c) If $\mathcal{D}_{f_{X}} \subset \mathcal{D}_{h}$ and $X_{*}=\emptyset$ then $\left|x^{k}\right| \rightarrow \infty$.

In certain applications it may be difficult to satisfy the descent requirement (7). Fortunately, it may be replaced by another condition on the tolerances $\left\{\epsilon_{k}\right\}$ (Kiwiel, 1995).

Theorem 5 Let $s_{k}=\sum_{j=1}^{k} t_{j}$ for all $k$. The assertions of Theorem 4 hold if the descent condition (7) is replaced by the accuracy condition $\sum_{k=1}^{i} s_{k} \epsilon_{k} / s_{l} \rightarrow 0$.

For choosing $\left\{\epsilon_{k}\right\}$, one may use the following result of (Kiwiel, 1995).

Lemma 6 (i) If $\epsilon_{k} \rightarrow 0$ then $\sum_{k=1}^{l} t_{k} \epsilon_{k} / s_{l} \rightarrow 0$ as $l \rightarrow \infty$. 
(ii) If $\sum_{k=1}^{\infty} \epsilon_{k}<\infty$ and $\left\{t_{k}\right\} \subset\left(0, t_{\max }\right]$ for some $t_{\max }<\infty$ then $\sum_{k=1}^{\infty} t_{k} \epsilon_{k}<\infty$.

(iii) If $\sum_{l=1}^{\infty} t_{l} / s_{l}=\infty$ and $\lim _{k \rightarrow \infty} \epsilon_{k} s_{k} / t_{k}=0$ then $\lim _{l \rightarrow \infty} \sum_{k=1}^{l} s_{k} \epsilon_{k} / s_{l}=0$.

(iv) If $\left\{t_{k}\right\} \subset\left[t_{\min }, t_{\max }\right]$ for some $0<t_{\min } \leq t_{\max }$ and $k \epsilon_{k} \rightarrow 0$ then $\sum_{k=1}^{l} s_{k} \epsilon_{k} / s_{l} \rightarrow 0$.

\section{LAGRANGIAN RELAXATIONS OF SET COVERING PROBLEMS}

Let $A \in\{0,1\}^{m \times n}, e=(1, \ldots, 1)^{T} \in \mathbb{R}^{m}$ and $c \in \mathbb{R}^{n}$. Consider the set covering problem

$\min \left\{c^{T} x: A x \geq e, x \in\{0,1\}^{n}\right\}$.

To solve its LP relaxation

$\min \left\{c^{T} x: A x \geq e, x \in[0,1]^{n}\right\}$

via the Bregman-prox method, let $f(x)=c^{T} x, X=\{x: A x \geq e\} \cap[0,1]^{n}$ and choose a B-function $h$ as in Ex. 3, so that $\mathrm{cl} \mathcal{D}_{h}=[0,1]^{n}$ and $\partial h=\{\nabla h\}$ on $\mathcal{D}_{\partial h}=(0,1)^{n}$. Then $\gamma^{k}=\nabla h\left(x^{k}\right)$ and $(8)$ reads

$x^{k+1} \approx \arg \min \left\{c^{T} x+\left[h(x)-\nabla h\left(x^{k}\right)^{T} x\right] / t_{k}: A x \geq e\right\}$.

The $d u a l$ problem, obtained by assigning a multiplier $\lambda$ to the constraints $A x \geq e$, is

$\max \left\{q(\lambda): \lambda \in \mathbb{R}_{+}^{m}\right\}$,

where $q$ is the concave continuously differentiable dual functional (Kiwiel, 1994)

$$
\begin{aligned}
g(\lambda) & =\min _{x}\left\{c^{T} x+\left[h(x)-\nabla h\left(x^{k}\right)^{T} x\right] / t_{k}+\lambda^{T}(e-A x)\right\} \\
& =-h^{*}\left(t_{k}\left(A^{T} \lambda-c\right)+\nabla h\left(x^{k}\right)\right) / t_{k}+e^{T} \lambda
\end{aligned}
$$

with $\nabla q(\lambda)=e-A x(\lambda)$ for all $\lambda \in \mathbb{R}^{m}$, where

$$
\begin{aligned}
x(\lambda) & =\arg \min _{x}\left\{c^{T} x+\left[h(x)-\nabla h\left(x^{k}\right)^{T} x\right] / t_{k}+\lambda^{T}(e-A x)\right\} \\
& =\nabla h^{*}\left(t_{k}\left(A^{T} \lambda-c\right)+\nabla h\left(x^{k}\right)\right) .
\end{aligned}
$$

Note that $q(\lambda)$ and $x(\lambda)$ are easily computed for $h$ as in Ex. 3 even in the large-scale case.

In effect, (12) may be solved approximately in the large-scale case by coordinate or block coordinate ascent (Kiwiel, 1994). However, having obtained an approximate solution $\lambda^{k}$ of (12), we cannot simply set $x^{k+1}$ to $x\left(\lambda^{k}\right)$, because $x\left(\lambda^{k}\right)$ will not, in general, be feasible in (11). The following result helps in constructing $x^{k+1}$ from $x\left(\lambda^{k}\right)$ to satisfy (4)-(6).

Theorem 7 Let $\lambda^{k}$ be an approximate solution of (12). Suppose $x^{k+1} \in \mathcal{D}_{\nabla h}$ satisfies $A x^{k+1} \geq e$ and $\left(A x^{k+1}-e\right)^{T} \lambda^{k}+e^{T} \delta_{+}^{k}-\left\langle\delta^{k}, x^{k+1}\right\rangle \leq \epsilon_{k}$, where $\delta^{k}=\left[\nabla h\left(x\left(\lambda^{k}\right)\right)-\right.$ $\left.\nabla h\left(x^{k+1}\right)\right] / t_{k}$ and $\left[\delta_{+}^{k}\right]_{i}=\max \left\{\delta_{i}^{k}, 0\right\}, i=1: n$. Then (4)-(6) hold with $\gamma^{k+1}=\nabla h\left(x^{k+1}\right)$. 
The algorithm of (Kiwiel, 1994) applied to (12) generates a sequence $\left\{\lambda^{(l)}\right\} \in \mathbb{R}_{+}^{m}$ s.t. $\left(A x\left(\lambda^{(l)}\right)-e\right)^{T} \lambda^{(l)} \rightarrow 0, x\left(\lambda^{(l)}\right) \rightarrow \hat{x}^{k+1} \in(0,1)^{n}$ and $\nabla h\left(x\left(\lambda^{(l)}\right)\right) \rightarrow \nabla h\left(\hat{x}^{k+1}\right)$ as $l \rightarrow \infty$, where $\hat{x}^{k+1}$ solves (11), so that $A \hat{x}^{k+1} \geq e$. Hence, for $l$ sufficiently large, one may take $\lambda^{k}=\lambda^{(l)}$ and obtain $x^{k+1}$ by increasing slightly certain components of $x\left(\lambda^{k}\right)$ (using $\left.A \in\{0,1\}^{m \times n}\right)$ to satisfy the conditions of Theorem 7 for any $\epsilon_{k}>0$. In effect, Theorem 5 applies, i.e., $\left\{c^{T} x^{k}\right\}$ converges to the optimal value of the LP relaxation (10), and $\left\{x^{k}\right\}$ converges to a solution of (10) if $\sum_{k} t_{k} \epsilon_{k}<\infty$.

Integer programming (IP) problems of the form (9) are usually solved by branch and bound, and their LP relaxations are often highly degenerate, which makes them difficult for simplex-based methods (Lindberg and Nöu, 1994). Our Bregman-prox method may be used not only for solving LP relaxations, but also for finding approximate solutions to the IP problem (9). Specifically, while solving the current dual subproblem (12), we may interpret $x_{j}\left(\lambda^{(l)}\right) \in(0,1)$ as the probability of having $x_{j}=1$ in a solution of $(9)$. Hence we may employ the randomized heuristic of (Lindberg and Nöu, 1994) for generating primal feasible solutions (PFS's).

Broadly speaking, our randomized PFS heuristic runs as follows.

- Start with $\check{x}=0$, i.e., all rows uncovered.

- While the set of uncovered rows $\mathcal{V}=\left\{i:(A \check{x}-e)_{i}<0\right\}$ is nonempty:

- Choose an uncovered row $i \in \mathcal{V}$.

- Choose $\hat{\jmath} \in\left\{j: A_{i j}=1\right\}$ at random, using the probabilities $x_{j}\left(\lambda^{(l)}\right)$. Set $\check{x}_{j}=1$.

- If possible, set $\check{x}_{j}=0$ for one or more $j$ s s.t. the final $\check{x}$ satisfies $A \check{x} \geq e$.

Of course, this heuristic may be implemented in various ways. The next section shows that it may produce points $\check{x}$ close to the primal integer optimum.

\section{NUMERICAL RESULTS}

We now report on preliminary numerical experience obtained with an implementation of our method programmed in Matlab. To save space, we only mention some of its features.

We chose $h$ as the Fermi/Dirac ' $x \log x$ ' entropy of Example 3.1, leaving other possibilities for future work.

The dual subproblems (12) were solved to relatively high accuracy by the coordinate ascent method of (Kiwiel, 1994). Each coordinate ascent employed a safeguarded Newton's scheme to reduce the directional derivative of $q$ by at least a quarter, i.e., the relaxation factor stayed in $[0.75,1]$, unless a bound became active. For simplicity, the coordinates (rows) were chosen cyclically. The randomized PFS heuristic was called once every three cycles (minor iterations), i.e., complete sweeps over the constraint set. We used constant proximal stepsizes $t_{k} \equiv 100 / \max _{i} c_{i}$ (with $c>0$ for our problems) and the initial accuracy tolerance $\epsilon_{1}=v_{\mathrm{LP}} / 10$, where $v_{\mathrm{LP}}$ was the (known) optimal value of the LP relaxation (10). The accuracy tolerances were decreased relatively slowly by setting $\epsilon_{k+1}=\kappa_{\epsilon} \epsilon_{k}$, where $\kappa_{\epsilon}=0.8$.

We present numerical results for a set of real-world set covering problems, emanating from airline crew scheduling problems (Wedelin, 1993). Table 1 highlights main features 
Table 1 Airline scheduling test problems

\begin{tabular}{lrrcrrr}
\hline Name & Rows & Columns & Density & \multicolumn{2}{c}{ NNZ } & \multicolumn{2}{c}{ IP-gap } \\
& & & $(\%)$ & col & row & \multicolumn{1}{c}{$(\%)$} \\
\hline B727 & 29 & 157 & 8.2 & 2.4 & 12.8 & 0.372 \\
ALITALIA & 116 & 1163 & 3.1 & 3.6 & 35.8 & 0 \\
A320 & 195 & 6927 & 2.4 & 4.6 & 163.1 & 0 \\
LH_A320 & 234 & 18752 & 1.9 & 4.4 & 351.4 & 0.003 \\
SASjump & 725 & 10353 & 0.57 & 4.1 & 58.9 & 0.025 \\
SASD9_2 & 1360 & 25026 & 0.33 & 4.4 & 81.5 & $\leq 0.018$ \\
\hline
\end{tabular}

Table 2 Convergence to the LP relaxation value

\begin{tabular}{lrrrr}
\hline Name & \multicolumn{4}{c}{ Relative accuracy (\%) } \\
& at minor iteration \\
& 10 & 25 & 50 & 100 \\
\hline B727 & 7.13 & 3.09 & 0.74 & 0.11 \\
ALITALIA & 1.12 & 0.54 & 0.13 & 0.03 \\
A320 & 9.91 & 0.98 & 0.37 & 0.23 \\
LH_A320 & 10.36 & 0.83 & 0.40 & 0.28 \\
SASjump & 7.85 & 2.15 & 0.66 & 0.41 \\
SASD9_2 & 6.77 & 1.72 & 0.70 & 0.16 \\
\hline
\end{tabular}

of these problems (NNZ stands for the mean number of nonzeros, and IP-gap for the difference in the optimal values of (9) and (10)). We may add that preprocessing has considerably reduced the size of these problems, and that CPLEX (version 2.1beta) could not solve the largest problem (Wedelin, 1993).

Table 2 shows the accuracy in the objective value relative to the (known) LP-value $v_{\mathrm{LP}}$ obtained after a given number of minor iterations (cycles). The numbers of major proxiterations were fairly small, e.g. , 15 for the ALITALIA problem. Anyway, most effort goes into minor iterations.

Table 3 shows the accuracy of the best primal solution obtained by the PFS heuristic relative to the (known) IP-value $v_{\mathrm{IP}}$ after a given number of sweeps ( $v_{\mathrm{IP}}$ is not known for SASD9_2). We note that the PFS heuristic was not called every cycle.

\section{REFERENCES}

Censor, Y. and Lent, A. (1981) An iterative row action method for interval convex programming. Journal of Optimization Theory and Applications, 34, 321-53.

Censor, Y. and Zenios, S.A. (1992) Proximal minimization algorithm with $D$-functions. Journal of Optimization Theory and Applications, 73, 451-64. 
Table 3 Convergence to the IP value

\begin{tabular}{llrrr}
\hline Name & \multicolumn{4}{c}{ Relative accuracy (\%) } \\
& 10 & 25 & 50 & 100 \\
\hline B7 minor & & & \\
& 10.54 & 6.67 & 3.50 & 0.64 \\
ALITALIA & 1.39 & 1.39 & 1.39 & 1.39 \\
A320 & 1.35 & 0.86 & 0.86 & 0.75 \\
LH_A320 & 2.13 & 1.64 & 1.64 & 1.64 \\
SASjump & 22.86 & 8.71 & 7.51 & 6.41 \\
SASD9_2 & 15.78 & 8.19 & 6.98 & 5.69 \\
\hline
\end{tabular}

Chen G. and Teboulle, M. (1993) Convergence analysis of a proximal-like minimization algorithm using Bregman functions. SIAM Journal on Optimization, 3, 538-43.

Eckstein, J. (1993) Nonlinear proximal point algorithms using Bregman functions, with applications to convex programming. Mathematics of Operations Research, 18, 202-26.

Kiwiel, K.C. (1994) Free-steering relaxation methods for problems with strictly convex costs and linear constraints. WP-94-89, International Institute for Applied Systems Analysis, Laxenburg, Austria, September. Revised version: July 1995.

Kiwiel, K.C. (1995) Proximal minimization methods with generalized Bregman functions. WP-95-24, International Institute for Applied Systems Analysis, Laxenburg, Austria, March.

Lindberg, P.O. and Nöu, A. (1994) Dual methods for large scale 0-1 problems. Technical report, Department of Mathematics, Royal Institute of Technology, Stockholm, April.

Rockafellar, R.T. (1970) Convex Analysis. Princeton University Press, Princeton, NJ.

Wedelin, D. (1993) Efficient algorithms for probabilistic inference, combinatorial optimization and the discovery of causal structure from data. Ph.D. thesis, Department of Computer Sciences, Chalmers University of Technology, Göteborg. 